\title{
Comparer des dessins de volumes en perspective
} cavalière

René Baldy et Jean-Francisque Chatillon

\section{(2) OpenEdition}

1 Journals

Édition électronique

URL : http://journals.openedition.org/trema/2404

DOI : 10.4000/trema.2404

ISSN : 2107-0997

Éditeur

Faculté d'Éducation de l'université de Montpellier

\section{Édition imprimée}

Date de publication : 1 décembre 1992

Pagination : 15-27

ISSN : 1167-315X

\section{Référence électronique}

René Baldy et Jean-Francisque Chatillon, « Comparer des dessins de volumes en perspective cavalière », Tréma [En ligne], 2 | 1992, mis en ligne le 01 décembre 1992, consulté le 19 avril 2019. URL : http://journals.openedition.org/trema/2404 ; DOI : 10.4000/trema.2404

Ce document a été généré automatiquement le 19 avril 2019

Trema 


\title{
Comparer des dessins de volumes en perspective cavalière
}

\author{
René Baldy et Jean-Francisque Chatillon
}

1 Si on analyse les processus qui sont derrière les performances observées (résultat et comportement), comme le fait systématiquement la psychologie cognitive, on est amené à étudier les processus responsables de l'organisation et du contrôle de la performance.

2 Ces processus sont divers. Certains relèvent des caractéristiques biomécaniques du système, d'autres des compétences cognitives dont dispose le sujet. L'ensemble des compétences cognitives dont dispose le sujet à un moment donné de son développement est lui-même complexe. Certaines requièrent que le sujet sémiotise des données utiles à l'exécution de la tâche, d'autres non. Les premières constituent une source d'organisation et de contrôle représentative de la performance. Les secondes constituent une source d'organisation et de contrôle non-représentative de la performance (par exemple, les indications prises directement sur le dispositif ou le contexte de la tâche peuvent être suffisantes pour assurer la réussite).

3 Si l'on veut analyser convenablement le rôle des processus représentatifs, il est tout à fait essentiel de distinguer les représentations mentales des représentations externes. Cette distinction qui n'est pas courante (Bresson, 1987; Denis, 1989) nous permettra, par exemple, de discuter le double statut des dessins d'objets et les difficultés de lecture qu'il est susceptible d'engendrer. Les problèmes que rencontrent des sujets adultes, dits de bas niveau de qualification (Pailhous et Vergnaud, 1989), pour lire et comparer des dessins de volumes en perspective seront interprétés dans le cadre de cette analyse. En conclusion quelques propositions didactiques seront avancées.

\section{1 - Représentations externes et représentations mentales}

4 Certaines propriétés de la réalité peuvent être traduites en langage naturel, ou en termes mathématiques faisant appel aux formalisations normalisées de l'algèbre ou de la théorie 
des ensembles par exemple. Ainsi, une équation est une représentation d'un phénomène physique au sens où elle s'y substitue pour les besoins du travail en cours. Les objets matériels peuvent être « représentés » par des maquettes en trois dimensions ainsi que par des figurations graphiques diverses tels que les plans, les schémas, les vues en perspective, le dessin technique... Les actions, les mouvements ou les relations entre objets peuvent être décrits par le geste, à l'aide d'organigrammes ou de programmes informatiques, par exemple. Dans ce cas, les représentations que traite l'ordinateur sont construites par le chercheur, et donc parfaitement définissables. Ces différentes représentations externes de la réalité possèdent chacune une syntaxe propre, plus ou moins bien adaptée à la présentation du contenu concerné. On admet généralement qu'il existe une analogie de fonction entre les représentations externes qui sont des produits matériels et les représentations mentales qui sont des produits cognitifs (cf. Bresson, 1987 ; Denis, 1989). Cela est vrai en partie. Les unes et les autres sont calculables, au sens de Vergnaud (1985, 1987, 1992), et il y a congruence entre leurs fonctions et leurs propriétés. Les mêmes propriétés rendent compte de la fonctionnalité des représentations mentales et des représentations externes. Les procédés sur lesquels reposent les figurations graphiques : simplification, sélection, déformation, etc. (cf., par exemple, Law, 1985) sont aussi ceux qui sont à l'œuvre dans l'élaboration des représentations mentales (Ochanine, 1981, Leplat, 1985). Les unes et les autres sont fonctionnelles en ce sens qu'elles offrent et supportent des possibilités d'actions plus efficaces aux individus qui sont capables de les élaborer et/ou de les exploiter. Cependant, plusieurs différences peuvent être soulignées et discutées.

Les représentations externes, et notamment graphiques, existent dans le monde matériel. Elles ont une existence et des propriétés facilement identifiables et analysables : ce sont des objets physiques que l'on peut voir et toucher. Par contre, on peut discuter leur vocation et leur capacité à représenter autre chose (et laquelle ?) qu'elles-mêmes. Aucun objet physique ne peut, en soi, désigner une chose plutôt qu'une autre (Putnam, 1984). Cette capacité de remplacement n'est pas directe, elle est surajoutée par le sujet. La ressemblance n'est d'ailleurs ni nécessaire, ni suffisante. Bresson (1987) note qu'il n'y a pas de représentation en soi, même dans le cas du fac similé. Dans le même ordre d'idée, Jacobi et Tonucci (1984) soulignent que l'image même la plus analogique peut ou veut signifier tout à fait autre chose que son référent. La correspondance entre un dessin ou une maquette et le monde réel doit toujours être construite par le sujet. Les observations de De Loache (1988), par exemple, montrent qu'avant trois ans environ, les enfants ne savent pas utiliser une maquette comme un substitut symbolique d'une réalité. Ainsi, l'interprétation et l'usage que le sujet peut faire d'une représentation externe dépend de ses capacités à l'assimiler comme un substitut symbolique d'une autre réalité. Ces capacités dépendent de son niveau de développement et de ses apprentissages spécifiques.

6 La psychologie cognitive s'intéresse, entre autres choses, à la façon dont les sujets codent mentalement les représentations externes. Les représentations mentales ne sont pas directement accessibles à l'observateur. Elles constituent toujours un "construct» hypothétique dont l'étude repose sur des observables ayant avec lui des rapports plus ou moins directs. La représentation que le psychologue infère est toujours une interprétation à justifier (cf. Le Ny, 1985, Leplat, 1985). Ainsi, on peut mettre en doute leur existence même (chez le bébé, chez l'animal, chez l'adulte) et discuter les propriétés qu'on leur prête. De ce point de vue, le choix des observables est important. Piaget et 
Inhelder (1947, 1963, 1966, par exemple) ont étudié la représentation de l'espace et les images mentales en analysant le contenu verbal ou graphique des productions des enfants. Shepard et Cooper (1982), Kosslyn (1980) étudient les propriétés des représentations mentales imagées en utilisant une technique chronométrique. Dans tous les cas, les propriétés des représentations du sujet doivent être "représentées » par le chercheur à partir d'indicateurs indirects, ce qui oblige à de nombreuses précautions méthodologiques. Comme le souligne Le Ny (1987), «établir des méthodes et des collections de données qui permettent d'effectuer de telles inférences, en diminuant les risques, mais sans atteindre la certitude, est probablement une des tâches les plus intéressantes qu'ait à résoudre la psychologie cognitive » (page 179). Mais, si l'on admet que les représentations mentales existent, alors leur vocation à permettre au sujet de se représenter le réel pour agir n'est pas discutée. Comme le dit Searle (1985), c'est une propriété de rez-de-chaussée de l'esprit.

\section{2 - Le double statut des dessins d'objets et les difficultés de lecture qu'il engendre}

7 Généralement, les représentations externes sont des expressions de représentations mentales. Elles sont de l'intelligence matérialisée, selon l'expression d'Oléron (1989). Elles sont des sources de données exploitables par l'individu pour élaborer des représentations nouvelles. Les représentations externes sont aussi des moyens de transmission de représentations mentales. Dans cette relation entre ces deux types de relation, le dessin d'objet possède un double statut. Le réseau de traits tracés sur la surface plane est un objet graphique autonome, c'est aussi une représentation externe d'un autre objet, réel ou imaginaire, présent ou absent. Dans ce cas, le dessin est un substitut symbolique qui renvoie, au delà de sa propre matérialité, à un autre espace et dont la lecture aboutit à une représentation mentale distincte des données visuellement perçues. Il est un objet culturel (artistique, technique, didactique, etc.), il peut constituer une mémoire externe fixant sur un support stable les représentations mentales. Lire un dessin d'objet, c'est extraire des données pour identifier l'objet et se représenter certaines de ses propriétés. Cette activité se présente comme une inférence sur l'objet à partir du dessin. Dans le cas de la lecture d'un dessin d'objet en perspective, le rapport dessin-objet dessiné, bien que largement analogique, s'appuie sur des règles de codage complexes et doit être reconstruit par le sujet pour que l'évocation de ce que représente le dessin soit possible. Les propriétés graphiques des dessins contribuent à déterminer le caractère bi - ou tridimensionnel de la lecture des dessins (Kopferman, 1930 ; Hochberg et Allister, 1953 ; Butler, 1982). Cependant, il y a dans tout dessin en perspective un aspect conventionnel beaucoup plus important qu'on ne le croit, et de nombreux résultats expérimentaux montrent que la différenciation entre le substitut symbolique et la réalité elle-même est source de difficultés importantes chez le jeune enfant (Von Schonen, 1974 ; Pierart et al., 1976), chez l'adulte qui apprend le dessin technique ou la géométrie (Rabardel et WeillFassina, 1987) ou qui vit dans une culture différente de la culture occidentale (Levy-Brulh, 1922 ; Bishop, 1983, Deregowski et Dziurawiec, 1986).

8 Cette analyse a sous-tendu une partie importante de notre travail sur les problèmes que rencontrent des sujets adultes, dits de bas niveau de qualification (Pailhous et Vergnaud, 1989), pour lire, comparer et exécuter des dessins de volumes en perspective cavalière. Ce travail était motivé par une question de terrain. Les moniteurs d'un centre de 
préformation avaient pour objectif pédagogique de compléter les connaissances des stagiaires afin que ces derniers puissent entrer en formation professionnelle pour adultes. Les contenus dispensés donnaient une grande place aux exercices destinés à entraîner les stagiaires à se représenter les propriétés spatiales des objets dessinés. Pour cela, ils utilisaient beaucoup les tâches de reconnaissance de formes. Par exemple, on proposait aux stagiaires de trouver parmi un ensemble de volumes dessinés en perspective cavalière celui qui correspond à un dessin modèle également en perspective cavalière. La figure 1 présente un exemple d'item.

Figure 1 - un exemple de tâche de reconnaissance

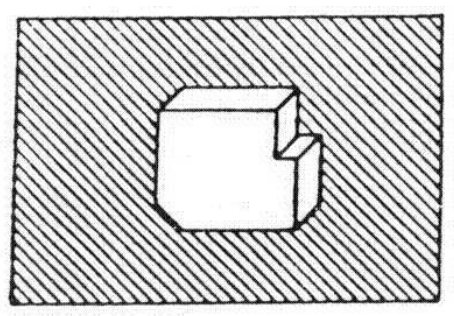

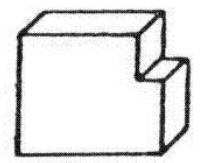

1

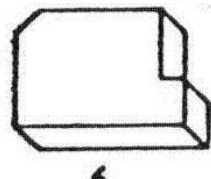

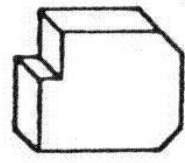

2

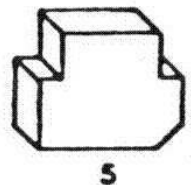

5

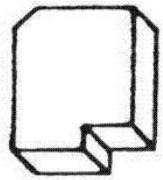

3

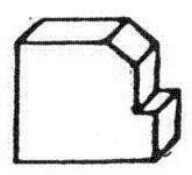

9 A un moment de leur pratique, les formateurs se sont interrogés sur le statut et l'intérêt de ce type d'exercices dans le contexte de la préformation d'adultes. Pour apporter quelques éléments de réponse à leur question, nous avons analysé ce que font les stagiaires dans ce type de tâches: lecture et comparaison de dessins d'objets en perspective, comparaison de dessins d'objets en perspective avec des objets réels, exécution en copie et de mémoire de dessins en perspective. Les résultats de ce travail sont interprétés en termes d'organisation et de contrôle représentatif de la performance.

\section{3 - Comparaison de volumes dessinés en perspective cavalière, par des adultes}

Les dessins proposés sont des gisements de données que le sujet exploite visuellement. Cette source d'organisation et de contrôle joue en permanence et en temps réel pendant l'exécution des comparaisons nécessaires pour trouver la réponse demandée. Les données visuelles participent aussi à la thématisation de la tâche et à la mise au point, par le sujet, d'une «référence représentée » (Chatillon, 1988) utilisable pour lire et comparer les 
dessins. Ainsi, dans des conditions normales d'exécution, le contrôle visuel se double d'un contrôle représentatif. Le contrôle représentatif devient essentiel quand les deux dessins à comparer ne sont pas simultanément présent dans le champ visuel et que l'un d'entreeux doit être mémorisé. Les propriétés de la référence représentée élaborée par le sujet dépendent, entre autres choses, de ce qu'il a lu sur les dessins. Certains peuvent procéder à une inférence représentative de type $:$ dessin $\rightarrow$ représentation du dessin, d'autres à une inférence de type: dessin $\rightarrow$ représentation du volume dessiné. Ces interprétations différentes de la tâche (comparer des dessins et comparer des volumes dessinés) soustendent des procédés d'exécution finalisés, organisés et contrôlés de façons différentes, et aboutissent à des résultats de niveaux et de structures différentes.

\section{Contrôle représentatif de la performance de type « dessin-dessin »}

Certains sujets redéfinissent la tâche de comparaison de volumes dessinés comme une tâche de comparaison de dessins. Ils ne différencient pas nettement, dans le dessin, son statut d'objet autonome de celui de substitut symbolique renvoyant à un autre objet et procèdent à une inférence représentative de type : dessin. Ce faisant, ils assimilent les dessins en perspective à des figures géométriques planes. Cette interprétation de la tâche dote les sujets d'une référence représentée pour l'exécution qui les conduit à rechercher deux dessins identiques. Ils travaillent dans l'espace de la page par comparaisons dessindessin. Leurs performances suggèrent qu'ils apprécient les dessins avec une référence représentée basée sur une représentation des dessins et non des volumes dessinés. On observe notamment que :

- ils réussissent globalement moins bien que les autres,

- ils réussissent mieux les « items dessins » dans lesquels le dessin modèle et le dessin bonneréponse sont superposables dans le plan que les « items volumes » dans lesquels le dessin modèle et le dessin bonne-réponse sont différents,

- quand ils se trompent, ils choisissent un dessin graphiquement proche du dessin modèle (Baldy et Chatillon, 1985 ; Baldy, 1986).

Cette interprétation est confirmée par les performances que les sujets produisent dans des tâches complémentaires. A la fin de la tâche de comparaisons de volumes dessinés, nous avons demandé aux sujets de reproduire le dessin (par exemple, celui présenté dans la figure 2) utilisé comme modèle (Baldy et Duval, 1988). 


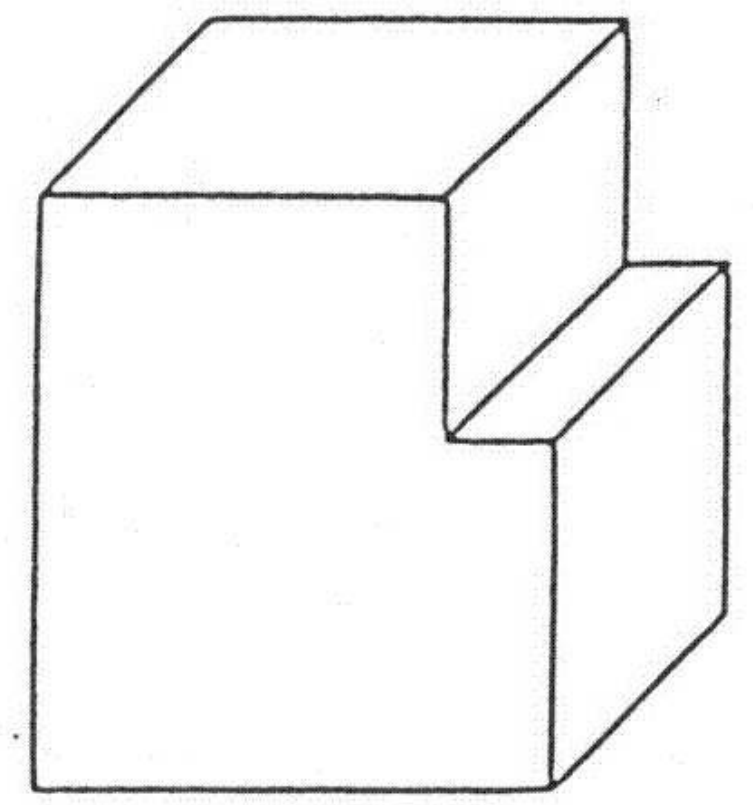

L'analyse de leur procédé d'exécution nous permet d'inférer certaines propriétés de la référence représentée avec laquelle ils travaillent. Cet ensemble de données montre qu'une partie des sujets, et notamment les moins performants dans la tâche de comparaison, exécute le dessin en perspective comme s'il s'agissait d'une figure plane : ils ne donnent pas un statut particulier à la vue de face et juxtaposent des parties ou remplissent un contour (la figure 3 donne des exemples de procédé d'exécution).

Figure 3 - Exemples de dessins réalisés par des sujets (les nombres portés sur les dessins décrivent le procédé d'éxécution)

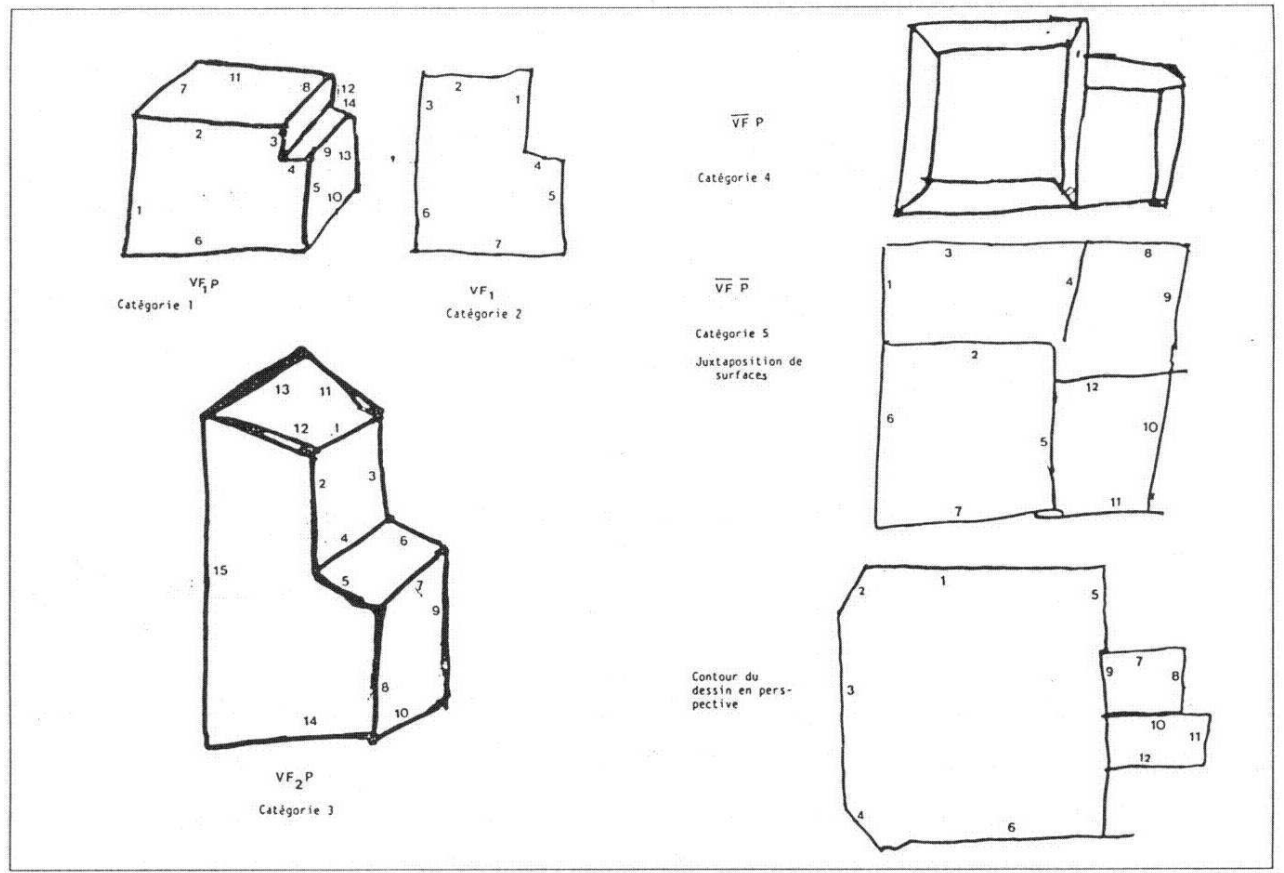


14 Nous avons demandé aussi à un groupe de sujets de rechercher parmi un ensemble de petits objets en bois, celui qui correspond à un dessin en perspective cavalière. Les résultats montrent (Baldy, 1988) que : soit l'inférence sur l'objet n'est pas effectuée du tout (le sujet choisit un objet plat de type « dessin en bois») soit le sujet l'effectue sur un objet familier qu'il peut nommer (escalier, meuble, par exemple) mais qui n'appartient pas à la classe des objets présentés. Ainsi, lorsque le sujet est engagé dans une tâche de comparaison d'objets dessinés avec un objet réel, ses possibilités d'exploiter sa présence sont limitées. Dans la tâche étudiée, le sujet dispose d'un ensemble de dessins d'objets en perspective placés sur chacune des pages d'un cahier, et d'un objet en bois servant de modèle. Le sujet doit décider si chaque dessin figure ou non le même objet que le modèle. On observe (Baldy, 1989) que la présence de l'objet réel n'aide pas les sujets à mieux reconnaitre les dessins, notamment ceux qui ont subi une rotation dans le plan de la feuille. Dans ce dernier cas, les résultats sont significativement moins bons que lorsque les dessins sont comparés à un dessin modèle.

Nous nous sommes demandé (Baldy et al., 1992) si les problèmes qu'ont certains sujets pour lire, comparer ou exécuter des dessins en perspective proviennent spécifiquement du traitement des rapports projectifs (perspective, point de vue) ou d'une "faiblesse fondamentale dans le domaine graphique » selon l'expression de Davies (1985, page 327). Pour apporter quelques éléments de réponse à cette question, nous avons comparé les performances (organisation du procédé d'exécution et propriétés du dessin produit) obtenues par un groupe de sujets dans deux tâches de dessins : la première consiste à reproduire une figure géométrique plane (tâche graphique n'exigeant pas le traitement de rapports projectifs), la seconde consiste à reproduire un dessin en perspective. Les résultats montrent que les problèmes rencontrés par les sujets proviennent essentiellement du traitement des rapports projectifs.

\section{Contrôle représentatif de la performance de type « volume-volume »}

16 Certains sujets interprètent convenablement la tâche. Ils considèrent les dessins comme des substituts symboliques d'objets réels, et procèdent donc à une inférence représentative sur le volume dessiné. Ils construisent, à partir des rapports spatiaux et conventionnels inscrits dans les dessins, une représentation mentale des propriétés spatiales des volumes. Cette interprétation de la tâche dote les sujets d'une référence représentée pour l'exécution qui les conduit à rechercher deux volumes identiques présentés, éventuellement, par deux dessins différents. Ils travaillent dans l'espace des volumes par comparaison volume-volume en procédant à des rotations mentales (au sens de Shepard et Metzler, 1971) et à des coordinations de points de vue (au sens de Piaget et Inhelder, 1947). Leurs performances montrent qu'ils apprécient les dessins avec une référence représentée basée sur une représentation des volumes dessinés. On observe notamment que :

- ils réussissent globalement mieux que les autres,

- ils réussissent de la même façon les « items dessins » et les « items volumes » (Baldy et Chatillon, 1985), et de la même façon que des sujets qualifiés (étudiants en section de technicien supérieur de mécanique). D'une façon générale, les comparaisons entre groupe de sujets de niveaux de formation différents (Baldy et Chatillon, 1985 ; Weill-Fassina et Vermersch, 1985, Cooper, 1990) suggèrent que ce facteur est déterminant dans les problèmes que les sujets rencontrent dans des tâches de cette nature. 
- quand ils se trompent, les erreurs qu'ils produisent traduisent les limites de leurs compétences représentatives par rapport aux exigences de la tâche (Baldy, 1986).

\section{Contrôle représentatif de la performance de type mixte}

Entre les deux groupes précédents, certains sujets ont conscience du double statut des dessins, sont finalisés sur la recherche de deux volumes identiques, mais rencontrent, cependant, des problèmes pour satisfaire aux exigences représentatives de la tâche. Ils parviennent à lire en trois dimensions les volumes dessinés, en attribuant, notamment, un statut particulier à la vue de face des dessins. Mais cette lecture ne se traduit pas par une représentation mentale des volumes suffisamment structurée pour supporter les transformations spatiales (rotations mentales et coordinations de points de vue) nécessaires aux comparaisons. Lorsque les exigences représentatives de la tâche dépassent ses possibilités d'exploitation de sa référence représentée, le sujet est contraint de revenir à des comparaisons dessin-dessin. Mais ce mode de contrôle du procédé d'exécution n'a pas le même statut que celui mis en œuvre par les sujets les plus faibles. Il est le moyen qu'ils utilisent pour résoudre les problèmes qu'ils rencontrent. On observe notamment que :

- certains sujets fondent leurs réponses sur la communauté de la vue de face entre les dessins,

- ils procèdent à des mises en relation de parties de dessins, et exploitent ainsi très bien la relation de symétrie lorsque le dessin réponse a subi, par rapport au modèle, une rotation dans le plan de $180^{\circ}$ (Baldy, 1986 ; Baldy et Duval, 1988).

\section{4 - Discussion}

Ce qu'apprend un sujet dépend de ce qu'il sait (ses connaissances antérieures disponibles) et de ce qu'il fait quand il exécute la tâche d'apprentissage, c'est-à-dire de la façon dont il exploite ses connaissances antérieures, notamment représentatives, pour organiser et contrôler ce qu'il y fait. Le sujet assimile la tâche (consignes, dispositif, etc.) à ses connaissances antérieures. Il lui attribue ainsi une signification dans la référence représentée qu'il met au point pour l'exécuter. Celle-ci détermine le but que le sujet se fixe, et éventuellement les moyens à mettre en œuvre pour l'atteindre Les propriétés de la référence représentée que le sujet va élaborer pour exécuter la tâche qu'on lui prescrit, fixent le résultat qu'il va rechercher, déterminent ce qu'il va faire, et par conséquent ce qu'il va apprendre.

Le rôle des tâches de lecture et de comparaison de dessins en perspective, sur lesquelles a porté notre travail de recherche, est d'apprendre aux sujets à se représenter les propriétés spatiales des volumes à partir de leurs dessins. On sait (Baldy et Chatillon, 1985 ; Baldy, 1986) que dans ces tâches, tous les sujets ne se fixent pas le même but. Un nombre important de sujets transforme la tâche prescrite de comparaison de volumes dessinés en une tâche effective de comparaison de dessins. Ces sujets apprécient les dessins avec une référence représentée qui est une représentation des dessins. Ils recherchent deux dessins identiques. D'autres sujets interprètent convenablement la tâche. Ils considèrent les dessins comme des substituts de volumes. Cette interprétation les dote d'une référence représentée qui les conduit à rechercher deux volumes identiques éventuellement présentés par deux dessins différents. Ces différentes façons d'exécuter la tâche ne présentent pas le même intérêt pour l'apprentissage visé. L'activité 
produite par les sujets qui comparent les dessins est faiblement formatrice de la représentation des volumes. Elle est même, par certains côtés, en opposition avec elle. Les sujets qui comparent les dessins doivent parvenir à se reformuler la tâche en terme de volume. Cette reformulation constitue la clé de l'apprentissage recherché. Elle permet au sujet de traiter la bonne version de la tâche. Même s'il produit beaucoup d'erreurs, il les produira dans le cadre d'une activité correctement finalisée. Pour que l'ensemble des sujets tirent profit d'un travail dans ces tâches, il convient de définir un dispositif et une progression qui conduisent tous les sujets à médiatiser leurs comparaisons par une représentation des volumes. Par exemple, les items caractérisés par un dessin modèle et un dessin bonne réponse superposables dans le plan de la feuille doivent être écartés, car on sait que cette propriété est exploitée par les sujets. Nous avons demandé (Baldy et al., à paraître), à des adultes, dits de bas niveau de qualification, de reproduire une figure plane et un dessin en perspective de complexité voisine (cf. figure 4).

Le dessin en perspective

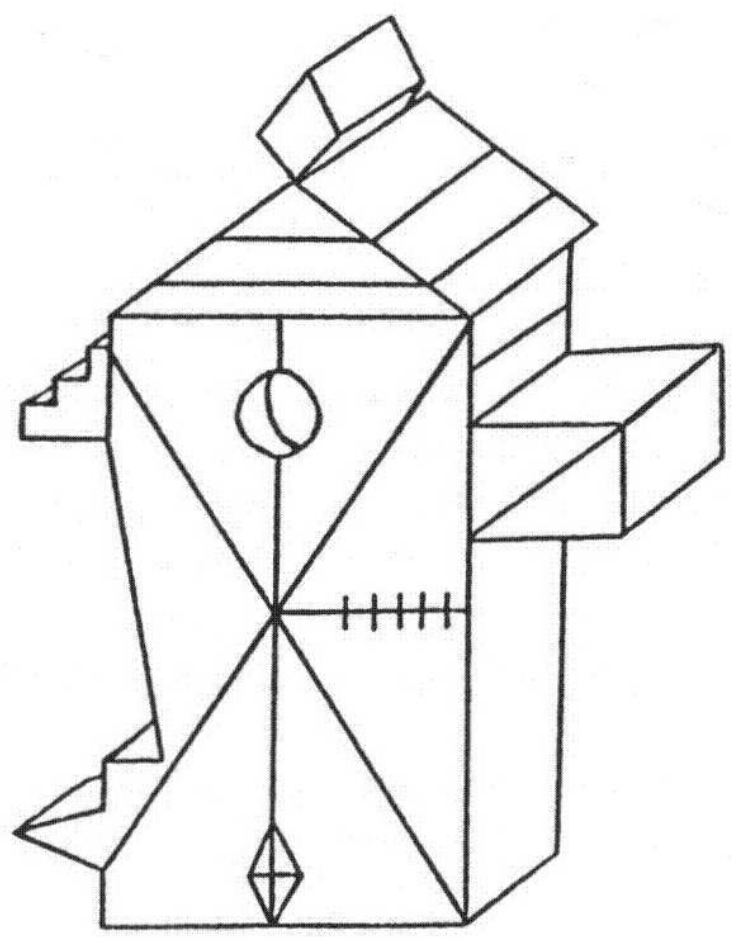




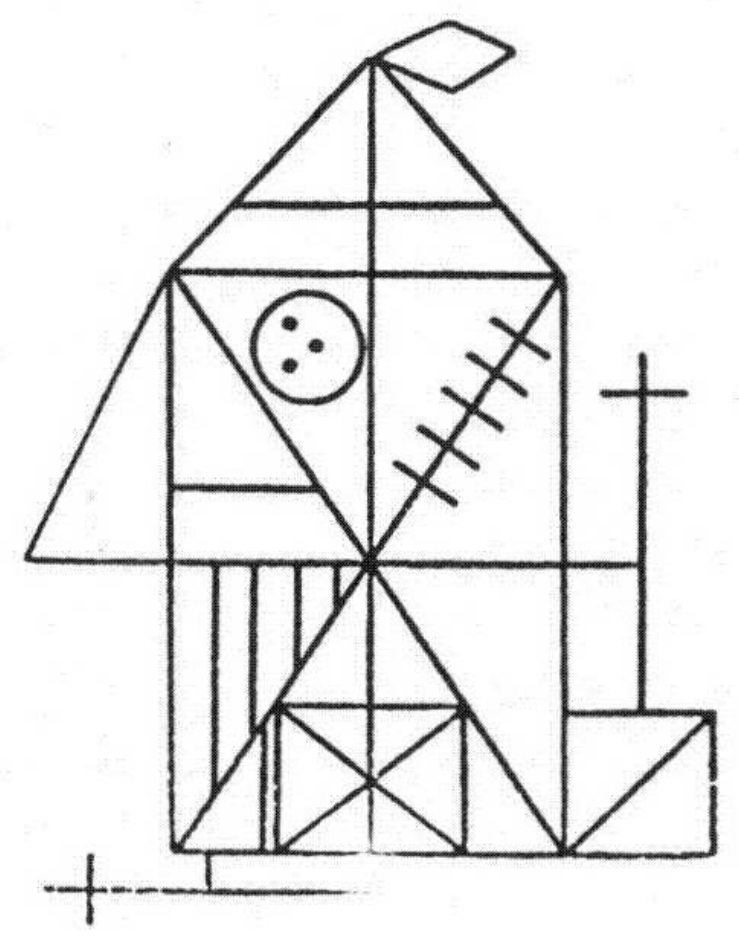

Un groupe de sujets commençait par l'exécution de la figure plane et terminait par l'exécution du dessin en perspective; un autre groupe procédait suivant l'ordre inverse. Nous avons étudié l'évolution de la structuration représentative des propriétés spatiales des deux dessins à travers l'analyse de l'organisation des procédés d'exécution. Les résultats obtenus montrent que les sujets qui exécutent la figure plane avant le dessin en perspective sont plus nombreux à organiser l'exécution de ce dernier que les sujets qui ont exécuté les dessins dans l'ordre inverse. Tout se passe comme si la structure spatiale apprise en exécutant la figure plane permettait aux sujets de mieux organiser l'exécution du dessin en perspective. Qu'est-ce qui est transféré ? Dans une certaine mesure, la vue de face du dessin en perspective peut être rapprochée de la figure plane que le sujet vient de dessiner. Il est possible alors que le sujet plaque sur le dessin en perspective l'organisation du procédé d'exécution construite sur la figure plane. Il est possible aussi, que la représentation de la figure plane dote le sujet de connaissances antérieures exploitables pour traiter les propriétés spatiales du dessin en perspective. Ce n'est plus alors simplement un procédé d'exécution qui est transposé, mais l'organisation représentative qui le sous-tend. Quelle qu'en soit sa nature ("procédural» ou «représentational», Novick, 1990) ce transfert présente un intérêt pédagogique. Il montre que l'organisation des propriétés projectives d'un dessin en perspective peut s'appuyer sur, et par conséquent être facilitée par, l'organisation topologique et euclidienne d'une partie du dessin (la vue de face) que le sujet a appris à reconnaitre. 


\section{BIBLIOGRAPHIE}

BALDY, R. (1986). Comparaisons de dessins de volumes en perspective cavalière par des adultes de bas niveau de formation. Archives de Psychologie, 54, 271-285.

BALDY, R. (1988). De l'espace du dessin à celui de l'objet. Une activité de mises en correspondance entre des dessins en perspective cavalière et des objets réels. Educational Studies in Mathematics, 19, 43-57.

BALDY, R. (1989). Comparaisons de dessins d'objets en perspective cavalière avec un dessin modèle ou avec un objet réel. European Journal of Psychology of Education, 4, 419-428.

BALDY, R., CHATILLON, J.F. (1985). La reconnaissance de dessins d'objets en perspective cavalière, les procédures développées par des adultes migrants dans des exercices de recherche de formes. Le Travail Humain, 48, 4, 307-320.

BALDY, R., DUVAL. J.(1988). Lecture, écriture et comparaison de volumes dessinés en perspective cavalière. Bulletin de Psychologie, 386, 617-624.

BALDY, R., CHATILLON, J.F., CADOPI, M., GAUSSERAND, V. (1992) Les problèmes rencontrés par des adultes de bas niveau de qualification pour lire et exécuter des figures planes et des dessins en perspective. L'Année Psychologique, 92, 559-579.

BALDY, R., CHATILLON, J., CADOPI, M. (à paraître). Dessin plan, dessin en perspective : étude des effets de transfert chez des adultes débutants. in : Graphismes d'espace, La pensée sauvage, Grenoble.

BISHOP. A.J. (1983) Space and geometry, in R. Lesh and Landau (Eds.), Acquisition of Mathematics Concepts and Processes, (pp. 175-203), New York : Academic Press.

BRESSON, F. (1987) Les fonctions de représentation et de communication, in PLAGET, J., MOUNOUD, P., BRONCKARD, J.P. (Eds.) Psychologie, (pp. 933-982) Paris : Gallimard.

BUTLER, D.L. (1982) Predicting the perception of three dimensional objects from the geometrical information in drauwings, Journal of Experimental Psychology. Human Perception and Performance, 8, 5, 674-692.

CHATILLON, J.F. (1988) La régulation représentative des actes complexes. Hypothèses et expériences, thèse de doctorat de psychologie. Université de Provence.

COOPER, L.A. (1990) Mental representation of three dimensionnal objects in visual problem solving and recognition, Journal of Experimental Psychology : Learning, Memory and cognition, 16, 6, 1097-1106.

DAVIES, T.N. (1985) An analysis of the depth cues in technical graphics, Le Travail Humain, 48, 321-330.

De LOACHE, J. (1988) L'enfant et le symbole, Pour la Science, n 132, page 13.

DENIS, M. (1989) Image et cognition. Paris : PUF.

DEREGOWSKI, J.B. \& DZIURAWIEC, S. (1986) Some aspects of comprehension of technical diagrams : An intercultural study. Le Travail Humain, 49, 1, 43-60.

HOCHBERG. J.E. et ALLISTER, A. (1953) A quantitative approach to figurai " goodness ». Journal of experimental psychology, 46, 361-364. 
JACOBI D. et TONUCCI. F. (1984) Concept de figurabilité et figurabilité des concepts dans le discours de vulgarisation scientifique (p. 209-216), in Signes et discours dans l'éducation et la vulgarisation scientifique, Sixièmes journées internationales sur l'éducation scientifique. A. Giordan et J.L. Martinans (Eds.) Université de paris VII.

KOPFERMAN, H. (1930) Psychologische Untersuchungen ùber die Wirkung zweidimensional Darstellungen korperlicher Gebilde, Psychologische Forschung, 13, 293-364.

KOSSLYN, S.M. (1980) Image and mind, Cambridge, MA : Hardvard University Press. LAW, J. (1985) Les textes et leurs alliés. Culture technique, 14, 58-69.

LE NY, J.F. (1985) Comment (se) représenter les représentations. In : S. Ehrlich (Ed.), Les représentations fonctionnelles. Psychologie Française, 30, 3, 4, 231-238.

LE NY, J.F. (1987) A quels risques peut-on inférer des représentations? in Signan (Ed) Comportement. Cognition, Conscience, la psychologie à la recherche de son objet, (pp. 161-179) paris : PUF.

LEPLAT, J. (1985) Les représentations fonctionnelles dans le travail, In : S. Eherlich (Ed.) Les représentations fonctionnelles. Psychologie Française, 30, 3, 4, 269-275.

LEVY-BRUHL (1922) La mentalité Primitive, Alcan : Paris.

NOVICK, L.K. (1990) Representational transfer in problem solving, Psychological Science, Vol 1, $\mathrm{n}^{\circ} 2$, OCHANINE, D. (1981) L'image opérative. Recueil de textes, Séminaire sur l'image opérative, Paris : Centre d'Education Permanente de l'Université de Paris I. OLERON, P. (1989) L'intelligence de l'homme, Paris : Armand Colin.

PAILHOUS, J. \& VERGNAUD, G. (1989) (sous la direction de) Adultes en reconversion, Paris : La documentation française.

PIAGET, J. \& INHELDER, B. (1947) La représentation de l'espace chez l'enfant, Paris : PUF.

PIAGET. J. \& INHELDER, B. (1963) Les images mentales in P. Oléron, J. Piaget, Inheiden B., \& P. Gréco (Eds), L'Intelligence. Traité de Psychologie expérimentale, Paris : PUF.

PIAGET, J. \& INHELDER, B. (1966) L'Image mentale chez l'enfant. Paris : PUF.

PIERAT, B., BIZET, A. \& COLLART, Y. (1976) Objets ou images ? Question méthodologique en psycholinguistique génétique, Archives de Psychologie, 44, 172, 267-276.

PUTNAM, H. Raison, vérité et histoire, Paris : Les éditions de Minuit.

RABARDEL, P. \& WEILL-FASSINA, A. (Eds.), (1987) Le dessin technique, Paris : Hermès.

SEARLE, J.R. (1985) L'Intentionalité, essai de philosophie des états mentaux, Paris : Les éditions de Minuit.

SHEPARD, R.N. \& METZLER. J. (1971) Mental rotation of three-dimensional objects. Science, 171, 701-703.

SHEPARD, R.N. \& COOPER, L.A. (1982) Mental images and their transformations Cambridge, MA : The MIT Press.

VERGNAUD, G. (1985) Concepts et schèmes dans une théorie opératoire de la représentation, Psychologie Française, 30, 3/4, 252.

VERGNAUD, G. (1987) Les fonctions de l'action et de la symbolisation dans la formation des connaissances chez l'enfant, in PIAGET, J., MOUNOUD, P., BRONCKARD, J.P. (Eds.) Psychologie, Paris : Gallimard.

VERGNAUD, G. (Ed.) (1992) Les sciences cognitives en débat, Paris : éditions du CNRS. 
VON SCHONEN, S. (1974) Etude de la lecture des représentations bi-dimensionnelles statiques en perspective projective chez des enfants de 2; 6 à 4 ; 5 ans, Archives de Psychologie, 42, 287-310.

WEILL-FASSINA, A. \& VERMERSCH, P. (1985) Un diagnostic opératoire dans des tâches de lecture de forme en dessin industriel : les cohérences des modalités de fonctionnement. Le Travail humain. 48.4, 341-358.

\section{RÉSUMÉS}

Nous étudions le rôle des processus représentatifs dans le contrôle de la performance dans des tâches de comparaison de dessins de volumes en perspective. Pour cela, nous distinguons les représentations mentales des représentations externes, nous analysons le double statut des dessins d'objets et les problèmes de lecture et d'écriture qu'il engendre. Les observations faites avec des sujets de bas niveau de qualification montrent que certains sujets assimilent les dessins en perspective à des figures planes et comparent les dessins et non les volumes dessinés. D'un point de vue didactique, cette activité est peu formatrice. Cependant, le travail sur des figures planes semble rendre plus facile l'apprentissage des propriétés projectives des dessins en perspective.

\section{AUTEURS}

\section{RENÉ BALDY}

Jeune Equipe Psychologie du Développement Cognitif, université Paul Valéry de Montpellier

\section{JEAN-FRANCISQUE CHATILLON}

Jeune Equipe Psychologie du Développement Cognitif, université Paul Valéry de Montpellier 